\title{
Sequential Array Cytometry: Multi-Parameter Imaging with a Single Fluorescent Channel
}

\author{
Daniel R. Gossett, ${ }^{1,2}$ Westbrook M. Weaver, ${ }^{1,2}$ Noor S. Ahmed, ${ }^{1}$ and Dino Di Carlo ${ }^{1,2}$ \\ ${ }^{1}$ Department of Bioengineering, University of California Los Angeles, Los Angeles, CA, USA; and ${ }^{2}$ California NanoSystems \\ Institute, Los Angeles, CA, USA
}

(Received 2 September 2010; accepted 19 October 2010; published online 7 December 2010)

Associate Editor Daniel Takashi Kamei oversaw the review of this article.

\begin{abstract}
Heterogeneity within the human population and within diseased tissues necessitates a personalized medicine approach to diagnostics and the treatment of diseases. Functional assays at the single-cell level can contribute to uncovering heterogeneity and ultimately assist in improved treatment decisions based on the presence of outlier cells. We aim to develop a platform for high-throughput, single-cellbased assays using well-characterized hydrodynamic cell isolation arrays which allow for precise cell and fluid handling. Here, we demonstrate the ability to extract spatial and temporal information about several intracellular components using a single fluorescent channel, eliminating the problem of overlapping fluorescence emission spectra. Integrated with imaging technologies such as wide field-of-view lens-free fluorescent imaging, fiber-optic array scanning technology, and microlens arrays, use of a single fluorescent channel will reduce the cost of reagents and optical components. Specifically, we sequentially stain hydrodynamically trapped cells with three biochemical labels all sharing the same fluorescence excitation and emission spectrum. These markers allow us to analyze the amount of DNA, and compare nucleus-to-cytoplasm ratio, as well as glycosylation of surface proteins. By imaging cells in real-time we enable measurements of temporal localization of cellular components and intracellular reaction kinetics, the latter is used as a measurement of multi-drug resistance. Demonstrating the efficacy of this single-cell analysis platform is the first step in designing and implementing more complete assays, aimed toward improving diagnosis and personalized treatments to complex diseases.
\end{abstract}

Keywords-Cytometry, Microfluidics, Single-cell, Immunocytochemistry, High content screening, Cellomics, Drug discovery, Personalized medicine, Point-of-care.

Address correspondence to Dino Di Carlo, Department of Bioengineering, University of California Los Angeles, Los Angeles, CA, USA. Electronic mail: dicarlo@ucla.edu,dicarlo@seas.ucla.edu

\section{INTRODUCTION}

High content screening (automated microscopy and image analysis) is a powerful tool for drug discovery, diagnostics, and biomedical research. ${ }^{1}$ Automated measurement of temporal and spatial information about targeted cellular processes can help elucidate drug-target interactions in vitro, ${ }^{10,22}$ identify the presence of rare abnormal cells in a tissue or blood, ${ }^{19}$ and enable high-throughput experimentation. ${ }^{1,4}$ Microscopy techniques are compatible with living cells, and working with biomolecules in their cellular microenvironment provides more accurate information about their function and molecular mechanisms. In contrast to microscopy techniques, bulk measurement tools such as plate readers or Western blots can produce misleading averages of populations and mask behaviors of rare abnormal cells or subpopulations. ${ }^{8}$ However, when performed manually, the throughput of single-cell microscopy measurements is low. Automation can increase the quantity of measurements and enhance reproducibility by limiting user bias. Current approaches to automation through robotics (used for high content screening) have been cost prohibitive and remain out of reach for point-of-care diagnostics, personalized medicine, and academic use. Further, the number of independent parameters that can be measured with these tools can be limited by overlap of fluorescence spectra. ${ }^{13}$ Immunophenotyping, for example indentifying T-cell subpopulations, stemness, or circulating cancer cells, often requires the identification of multiple biochemical parameters. ${ }^{2,3,13,19}$ Moreover, dynamic processes such as drug permeability through a cell monolayer may be best characterized by a temporal parameter. ${ }^{15,20}$ While there is much effort toward expanding the capabilities of the scanning microscope and other currently used 
cytometric methods, such as flow cytometry, ${ }^{3,17}$ several other tools and techniques are being developed which aim to bring down cost and expand access through miniaturization and simplification. High-throughput, parallel fluorescence detection has been achieved by an integrated zone-plate array. ${ }^{18}$ Fiber-optic array scanning technology can scan substrates 500 times faster than conventional scanning micrscopy. ${ }^{12}$ Wide fieldof-view lens-free fluorescent imaging on a chip is another alternative to mechanical scanning lens-based systems. ${ }^{6}$ This compact technology has achieved $\sim 10 \mu \mathrm{m}$ spatial resolution over an $8-\mathrm{cm}^{2}$ field of view with a single image. ${ }^{5}$ Many of these techniques are well equipped to identify rare, single-cell events. However, positive and negative identifications may require a composite overlay of several signals demonstrating colocalization and some of these techniques are currently limited to a single wavelength. In this study we propose a technique called "Sequential Array Cytometry" which will release these tools from these restrictions.

Sequential array cytometry employs microfluidic hydrodynamic trapping to create massive arrays of single cells (Fig. 1) which could be integrated with automated microscopy or some of the massively parallel or wide field-of-view detection schemes or imagers which have recently been reported. While there exist numerous methods of cell trapping ${ }^{14}$ or patterning methods, hydrodynamic traps were selected due to their simplicity, ease of implementation, potential integration with optical and fluid components of other cytometric or cell imaging methods, and their ability to handle suspended, uncultured cells in a gentle manner. ${ }^{9}$ We have previously characterized these traps and demonstrated their efficacy in immobilizing cells for single-cell enzyme kinetics. ${ }^{7}$ Holding the suspended cells in place while probing them with biochemical fluorescent indicators we can extract quantitative data which can be fit to kinetic models. While the cells are immobilized we can exchange the solutions around the cells and probe with multiple stimuli or biochemical stains. Further, we hypothesized that sequential stains could be of the same fluorescent excitation and emission spectra as we could quantify the difference in fluorescence levels to obtain the increase due to a single parameter. As such we would not be limited by the number of filters or spectrum overlaps of available fluorophores and could extract quantitative and dynamic information from a number of parameters. An example of a dynamic event is a permeability assay whereby drug efflux from cells is monitored with time. The rate of efflux, an indicator of multidrug resistance (MDR) has been used to study the effectiveness of MDR inhibitors and substrates in increasing drug toxicity. ${ }^{11,15,20}$ Here, we demonstrate the temporal aspect of sequential array cytometry by quantifying the efflux of fluorescent indicators from hydrodynamically trapped Caco-2 cells.
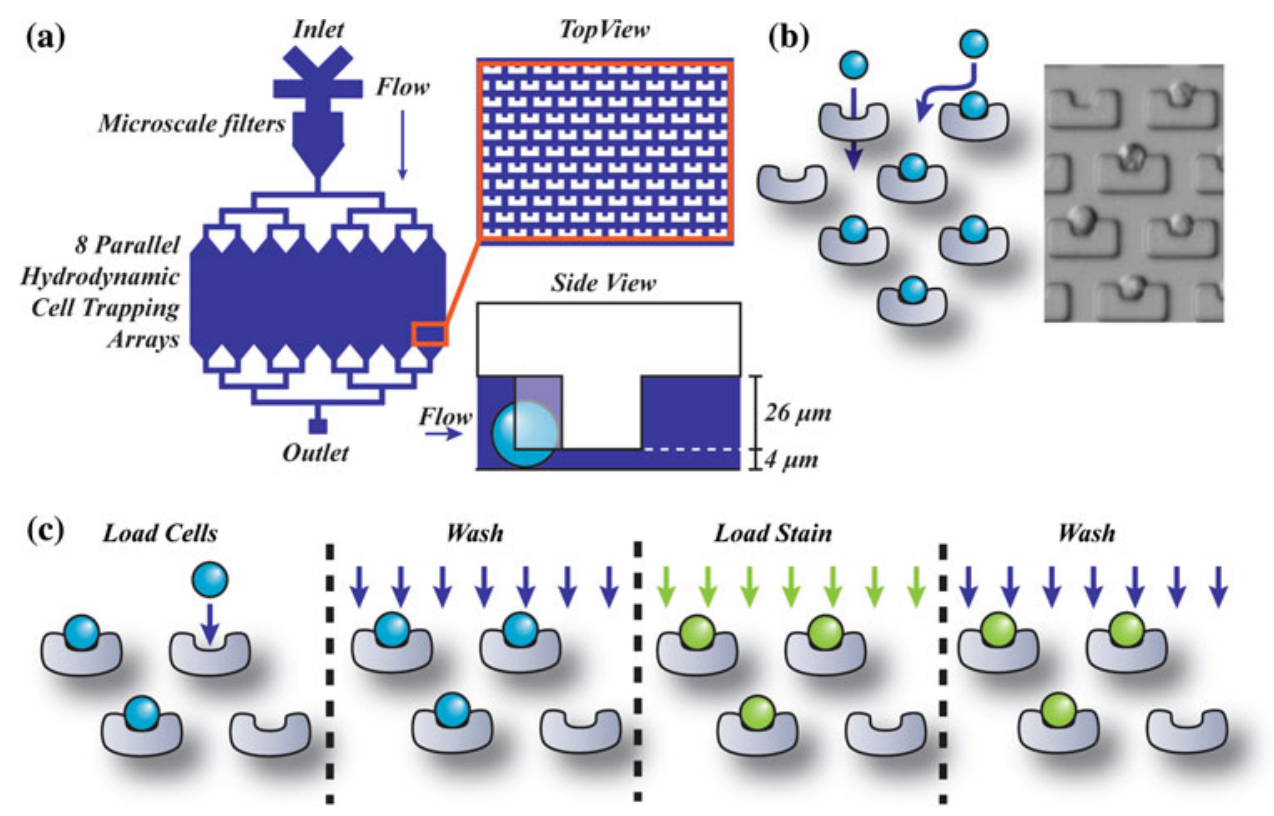

FIGURE 1. Hydrodynamic cell trapping for exchange of solutions and imaging. (a) Three-dimensional hydrodynamic cell traps were created in massive arrays (previously reported by Di Carlo et al. ${ }^{9}$ ). (b) Cell traps are raised to allow fluid streamlines to pass beneath them, dragging in cells. No external forces other than the fluid driving force are needed. (c) Hydrodynamically trapped cells can have fluid solutions exchanged around them, allowing for sequential staining and imaging of a constant set of cells. 


\section{MATERIALS AND METHODS}

\section{Microfluidic Channel Fabrication}

Microfluidic channels were fabricated by replica molding. ${ }^{9,21}$ In brief, a two-layer mold was constructed in SU-8 50 (MicroChem Corp., Newton, MA, USA) using standard photolithographic methods. A transparent, elastomeric polymer, polydimethylsiloxane (PDMS; Sylgard 184 Silicone Elastomer, Dow Corning Corp., Midland, MI, USA), was cast over the mold and cured at $65{ }^{\circ} \mathrm{C}$ for at least $3 \mathrm{~h}$. The PDMS could then be removed from the mold and inlet and outlet holes were punched with a pin vice assembly (Technical Innovations, Inc., Angleton, TX, USA). The molded side of the PDMS and a slide glass were activated with air plasma for $30 \mathrm{~s}$ then placed in contact to form a permanent bond. Minimal pressure was applied to ensure contact, but care was observed to not collapse raised channel features onto the glass. The latter would prevent necessary flow through the hydrodynamic cell traps. The completed channels were left to bake at $65^{\circ} \mathrm{C}$ for at least $5 \mathrm{~min}$ prior to use. Later, the inlet and outlet of the channel could be fitted with polymeric tubing and have fluid driven through them by a syringe pump. The top surface of the PDMS was sterilized with $70 \%$ ethanol then washed with sterile water.

\section{Solutions and Cell Lines}

The fluorescent stain solutions used were $2 \mu \mathrm{M}$ calcein AM (Invitrogen Corporation, Carlsbad, CA, USA), $1.5 \mu$ M SYTO 16 green fluorescent nucleic acid stain (Invitrogen), and $25 \mu \mathrm{g} / \mathrm{mL}$ FITC-conjugated Lectin from Triticum vulgaris (wheat germ agglutinin; Sigma-Aldrich Corp., St. Louis, MO, USA). $100 \mu \mathrm{M}$ fluoxetine (Cerilliant Corporation, Round Rock, TX, USA) was used as an inhibitor of drug-resistance transporters. The HeLa cell line (epithelial; human cervical adenocarcinoma) was propagated in Dulbecco's Modification of Eagle's Medium (DMEM) $1 \times$ with L-Glutamine, $4.5 \mathrm{~g} / \mathrm{L}$ Glucose and Sodium Pyruvate, $10 \%$ fetal bovine serum (FBS), and 1\% Penicillin/ Streptomycin at $37{ }^{\circ} \mathrm{C}$ and $5 \% \mathrm{CO}_{2}$. The Caco-2 (epithelial; human colorectal adenocarcinoma) cell line was propagated in DMEM $1 \times$ with L-Glutamine, $4.5 \mathrm{~g} / \mathrm{L}$ Glucose and Sodium Pyruvate, 20\% FBS, and 1\% Penicillin/Streptomycin at $37{ }^{\circ} \mathrm{C}$ and $5 \%$ carbon dioxide $\left(\mathrm{CO}_{2}\right)$. For sequential array cytometry, both cell lines were released from tissue culture flasks with $0.25 \%$ Trypsin and resuspended in their culture media. For measurements of noise and saturation limit $9.9 \mu \mathrm{m}$ fluorescent polymer microspheres (Thermo Scientific, Waltham, MA, USA) were used.

\section{Hydrodynamic Cell Trapping and Solution Exchange}

Channels (see Fig. 1) were prefilled with $1 \times$ phosphate buffered saline (PBS) by injecting PBS through the outlet (outlet during the rest of the protocol) with a syringe pump at a flow rate of $20 \mu \mathrm{L} / \mathrm{min}$. The inlet was obstructed with a metal pin during this time to eject air through the air-permeable PDMS walls. Subsequently, the pin was removed, and a $20-\mu \mathrm{L}$ drop of PBS was loaded over the inlet. The flow direction on the syringe pump was reversed such that fluid was drawn from the drop through the channel. The drop was replenished before any air could enter the inlet. For cell loading, a suspension of cells was added dropwise to the inlet and the flow rate was reduced to $10 \mu \mathrm{L} / \min$ (optimized for cell trapping). For all other solutions and wash steps $10 \mu \mathrm{L} / \mathrm{min}$ was used.

\section{Image Capture Post-Processing}

Channels were mounted on an inverted microscope stage and secured. The appropriate axial position and exposure times for the selected stains were chosen prior to experiments. Ambient light was minimized. Grayscale fluorescent images were captured every $30 \mathrm{~s}$ using an automated time-lapse function in the image acquisition software (NIS Elements; Nikon Instruments Inc., Melville, NY, USA). In-between exposures the shutter was closed to minimize bleaching. Image processing was carried out in NIS Elements and ImageJ (http://rsbweb.nih.gov/ij/). The final image of the calcein AM staining process was used to identify the address and size of every cell within the imaged portion of the array. The mean intensity at every address in defined regions of interest (ROIs), which encompass the cell area at each address, was stored over time for kinetics experiments. For multi-parameter image reconstruction an image was selected from each staining step. The images were opened in ImageJ. Using the "Image Calculator" function sequential images were subtracted from one another to obtain only the staining that occurred during that interval. These were pseudo-colored different colors using the "Lookup Tables" function and overlayed in NIS Elements.

\section{Statistics}

As stated above, the mean intensity per area for each cell was extracted from the images at every time point. A two-tailed $t$-test assuming unequal variances was carried out comparing the mean of control and experimental conditions. 


\section{RESULTS}

The number of independent parameters that can be measured within a single fluorescent channel is limited by the noise and the number of grayscale levels before saturation for the detection scheme. We hydrodynamically trapped non-bleaching fluorescent polymer microspheres, measuring their mean fluorescent intensity per area every $30 \mathrm{~s}$ for $3 \mathrm{~min}$ (experimental conditions identical to subsequent cell-based assays) at nine exposure times from 5 to $45 \mathrm{~ms}$. As seen in Fig. 2a the mean fluorescent intensity per area $(N=8$ particles) increases with exposure time until the sensor's limit is reached, defining the saturation limit (Mean $\approx 9300 \mathrm{AU} / \mu \mathrm{m}^{2}$ ). The maximum standard deviation of the mean, $\sigma$, in this range of exposure times was $39 \mathrm{AU} / \mu \mathrm{m}^{2}$. A practical definition of the noise is $4 \sigma$; thus staining increments should be larger than $4 \sigma$ to unambiguously resolve these levels above background noise (Fig. 2b). For our experiments each staining procedure resulted in an intensity increment greater than $1000 \mathrm{AU} / \mu \mathrm{m}^{2}$.
To demonstrate the feasibility of sequential array cytometry we stained three independent components of the ubiquitous $\mathrm{HeLa}$ cell line (hydrodynamically trapped within an array) with all fluorescein spectrum dyes and extracted the contribution to the fluorescent signal from each molecular probe by image subtraction. As described above, fluorescent images are recorded throughout the program with the shutter closed in between images to prevent bleaching. The important frames are those where fluorescence intensity has become level; these occur during the wash steps after each stain (Fig. 3a). One frame from each wash step was selected for the image calculations. Calcein AM is cleaved by internal carboxyl esterases to fluorescent calcein and is typically used as a live cell indicator as it diffuses through the plasma membrane. It is located throughout the cytosol (Fig. 3a, left). A FITCconjugate of wheat germ agglutinin binds glycoconjugates, typically found on the surface of the cell. Lastly, SYTO 16 stains nucleic acids in the nucleus and mitochondria. The calculated differences between fluorescence intensities between these images reveals

(a)
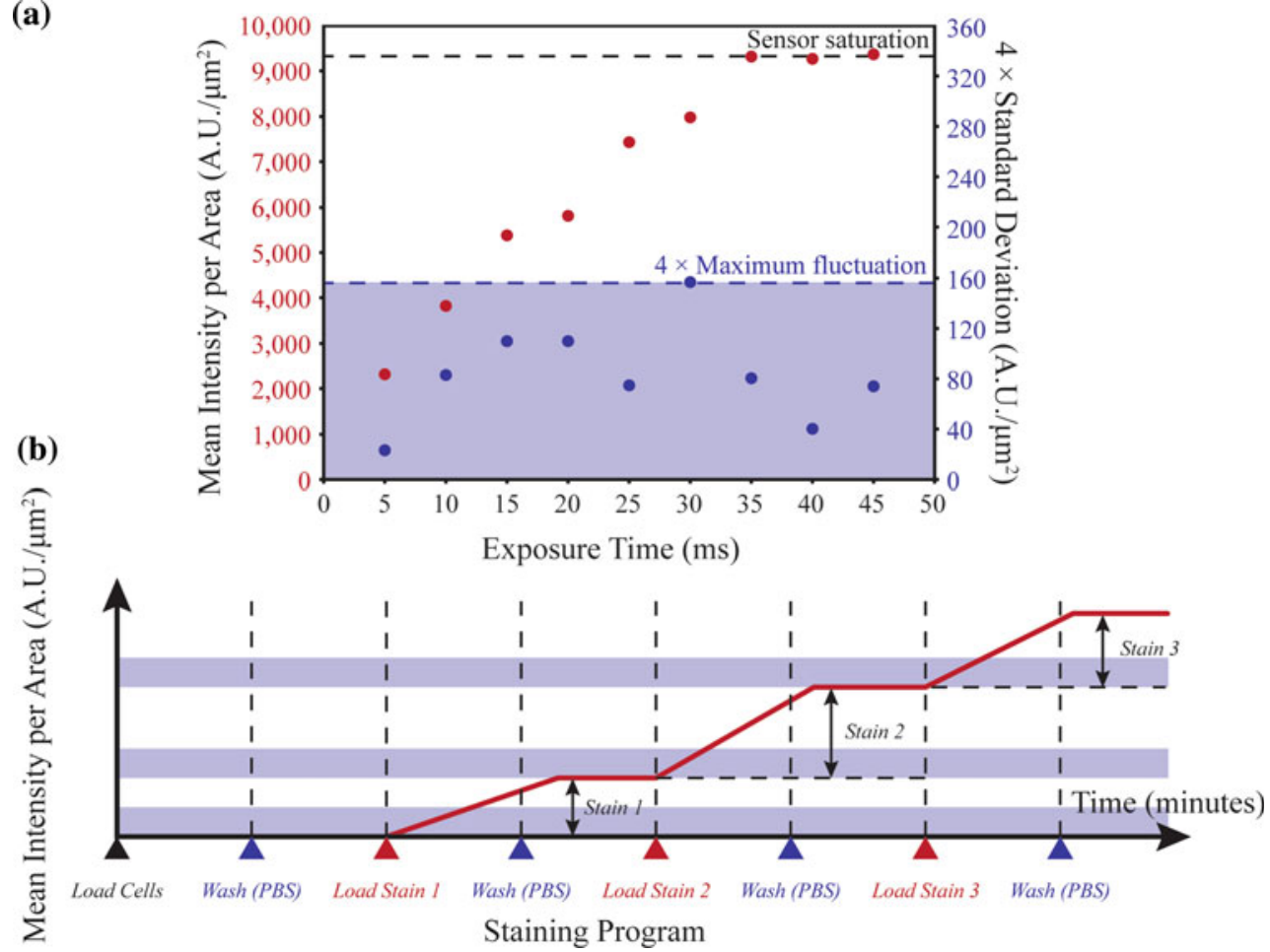

FIGURE 2. Principles of sequential array cytometry. (a) The fluorescence intensity (mean intensity per area) of non-bleaching $9.9 \mu \mathrm{m}$ fluorescent polymer microspheres $(N=8)$ captured every $30 \mathrm{~s}$ for $3 \mathrm{~min}$ increases with exposure time. Four times the standard deviation $(4 \sigma)$ of the mean intensity per area is a practical measurement of noise or fluctuations. Hypothetically, the mean intensity per area when the sensor is saturated divided by $4 \sigma$ is the number of independent parameters that could be measured with sequential array cytometry with careful planning of exposure time and label concentration. (b) A staining program is devised such that hydrodynamically trapped cells undergo staining with dyes that share fluorescence excitation and emission spectra and increase the mean intensity per area greater than $4 \sigma$ (indicated by blue horizontal bars). The fluorescence is quantified so that the difference between intensity levels can be attributed to one stain. The shutter is closed between exposures to ensure bleaching does not adversely affect the calculations. 
what was contributed by the subsequent stain (Fig. 3b). These new images were adjusted for brightness and pseudo-colored, then overlayed to create what one would typically expect from a multi-fluorophore staining protocol (Figs. 3c and 3d).

To characterize the dynamics of drug transporters at the single-cell level we trapped Caco-2 cells, perfused with calcein AM, and quantified the increase in emitted fluorescence with time. This rate is reduced by efflux of calcein AM by MDR transporters such as MDR-1. In
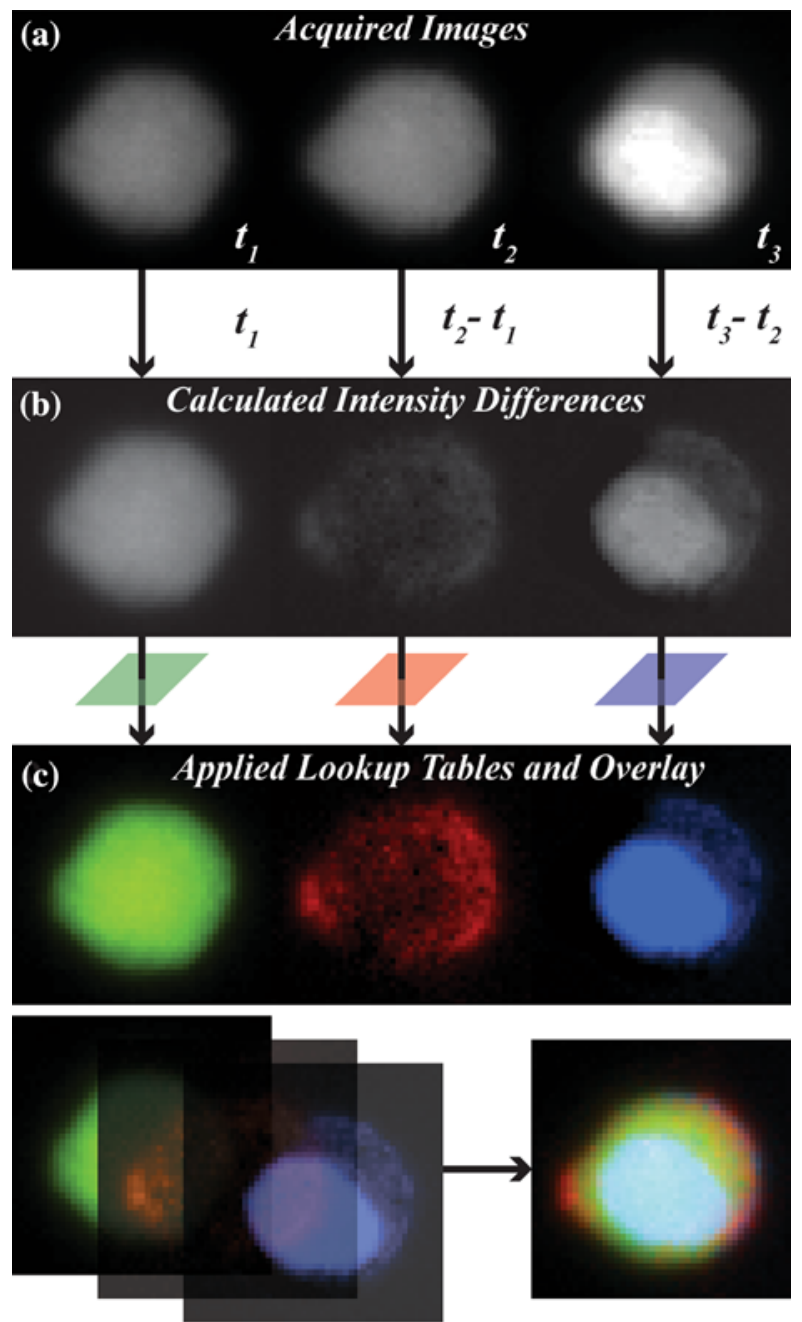

(d)

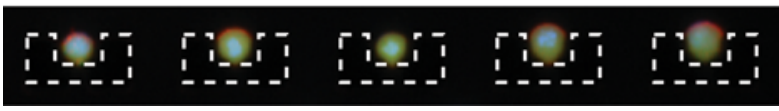

FIGURE 3. Demonstration of sequential array cytometry. (a) Selected grayscale quantitative fluorescent microscopic images $(10 \times$ objective; pixels expanded $1000 \%)$ of a cell acquired over time with a single filter. Before each image a different biomolecule stain was added to the hydrodynamically trapped cells. (b) Image calculations performed with ImageJ. (c) The resulting images were pseudo-colored. Their intensity was scaled and then they were overlayed in NIS elements for a composite multiparameter image. (d) A row of arrayed cells prepared with sequential array cytometry. a separate condition we first exchanged the solution around the cells to $100 \mu \mathrm{M}$ fluoxetine, a known inhibitor of these transporters, ${ }^{16}$ then replaced it with $2 \mu \mathrm{M}$ calcein $\mathrm{AM} / 100 \mu \mathrm{M}$ fluoxetine. The single-cell fluorescence increase was quantified over $8.5 \mathrm{~min}$ (Fig. 4). Statistical tests, as described in the section "Materials and methods," demonstrate statistical significance of the difference between inhibited $(N=232)$ and uninhibited populations $(N=112)$ after $4 \mathrm{~min}$. A histogram shows the distribution of single-cell data, identifying a non-normal distribution. As rare cells can dominate clinical outcomes (e.g., rare drug-resistant cells leading to cancer relapse) the ability to identify a whole population distribution is critical but cannot be achieved with bulk measurements. The portion of the

(a)
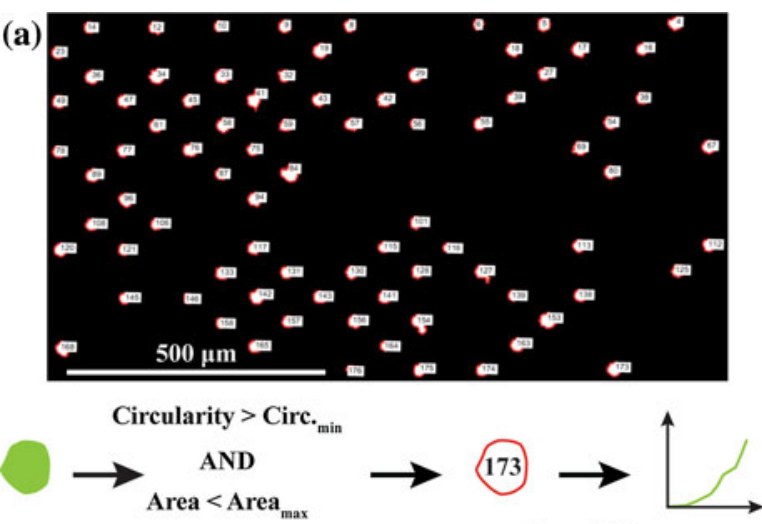

Threshold Image

Define ROI

Record Mean Intensity/Area

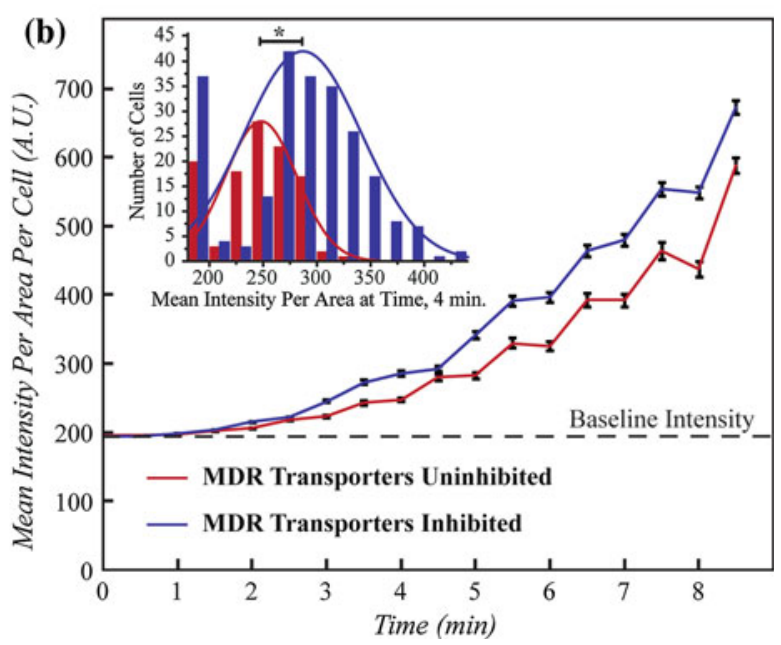

FIGURE 4. Dynamic molecular events can be monitored simultaneously with quantitative biomarkers using sequential array cytometry. (a) A threshold was applied to images to discount debris and cell aggregates then an ROI was defined for the cell area. The mean intensity per area was recorded at every time point. (b) The means for the inhibited and uninhibited populations were plotted against time (error bars are standard error of the mean). A histogram shows the distribution of mean intensities per area after 4 min (inset). The experimental condition has a significantly larger mean than the uninhibited control $\left({ }^{*} p<0.001\right)$. 

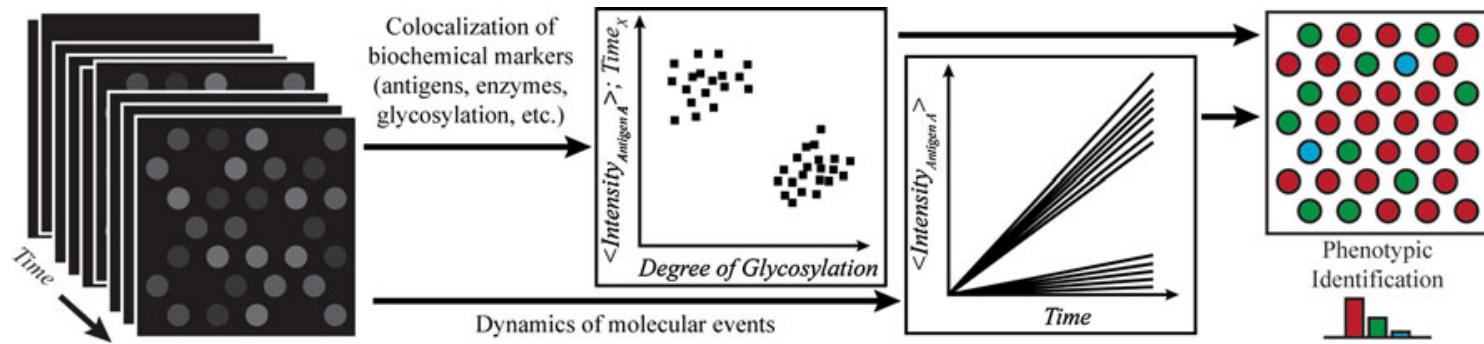

FIGURE 5. Sequential array cytometry uses a sequence of images obtained at a single wavelength to provide quantitative measures of intracellular and surface biomarkers as well as measures of dynamic molecular events which in combination has the potential to be used to more accurately establish phenotype or molecular mechanisms.

cells at the baseline intensity can be removed by software as they were late to enter the trapping region. While the dynamics of drug efflux are not thoroughly used here, we have the ability to extract kinetic information after having already stained the cell (i.e., when the cells do not share the same baseline intensity values).

\section{DISCUSSION}

Low cost cytometric instruments for personalized medicine and point-of-care diagnostics will require simplified optics and operation speeds faster than current state-of-the-art automated microscopy and flow cytometry. Even these powerful instruments can be limited by the overlap of fluorescence emission spectra while some of the new imaging techniques for these applications are currently limited to a single wavelength. Here, we present a method which removes both of these limitations. We demonstrate the ability to create composite images, but more importantly the ability to identify colocalized parameters in a way that is not limited by spectral overlap. It remains to be shown the number of independent parameters that can be obtained by sequential array cytometry, but the theoretical limit with our current optical setup is approximately 60 components (our measured saturation number of levels divided by $4 \sigma$ ). It will depend on the dynamic range of the detection scheme, the specificity of fluorescent probes, the quality of solution exchange, and the length of time that cells remain intact while trapped. The photostability of fluorescent probes should be considered when designing sequential array cytometry protocols; the shutter is open only briefly (milliseconds to seconds) at each exposure time to minimize photobleaching, but over long periods of time bleaching of an unstable stain could result in inaccuracies if not taken into account. Alternatively, after imaging, bleaching could be actively used to drastically reduce the intensity and noise contribution of the previous stain, enabling a further increase in the number of stains possible.
There are some especially promising combinations of sequential array cytometry with emerging imaging techniques. The immense field-of-view of lens-free fluorescent imaging on a chip ${ }^{6}$ or other wide-field imaging technologies coupled with the ability to measure numerous biochemical parameters in a single experiment in a massive, parallel array of cells would be a very powerful tool for biomedicine. It could also be cost-effective for academic studies, with greater sample size enhancing statistical accuracy. The ability to distinguish between free and bound fluorescent probes, provided by imaging using lenses that focus light on the sample, may be sacrificed by this type of integration, but our ability to rapidly exchange solutions, depleting free probes, may mitigate this problem. In this study, we have introduced another way of handling the probe and wash solutions (dropwise addition coupled with a negative pressure). This could be adapted to industrystandard, automated fluid handling systems for microtiter plates. In addition to binary or quantitative measures of biochemical markers, sequential array cytometry enables measurements of dynamic molecular events. These complementary measures will be useful for phenotypic identification (Fig. 5), determining molecular mechanisms, and drug permeability assays. And, techniques like sequential array cytometry and widefield imaging technologies have the potential to make these measures available for personalized medicine.

\section{ACKNOWLEDGMENTS}

We thank Aydogan Ozcan and Ahmet Coskun for helpful discussions and Henry T. K. Tse for assistance with automated data processing.

\section{OPEN ACCESS}

This article is distributed under the terms of the Creative Commons Attribution Noncommercial License which permits any noncommercial use, 
distribution, and reproduction in any medium, provided the original author(s) and source are credited.

\section{REFERENCES}

${ }^{1}$ Abraham, V. C., D. L. Taylor, and J. R. Haskins. High content screening applied to large-scale cell biology. Trends Biotechnol. 22:15-22, 2004.

${ }^{2}$ Adewumi, O., et al. Characterization of human embryonic stem cell lines by the international stem cell initiative. Nat. Biotechnol. 25:803-816, 2007.

${ }^{3}$ Chattopadhyay, P. K., et al. Quantum dot semiconductor nanocrystals for immunophenotyping by polychromatic flow cytometry. Nat. Med. 12:972-977, 2006.

${ }^{4}$ Cheong, R., C. J. Wang, and A. Levchenko. Using a microfluidic device for high-content analysis of cell signaling. Sci. Signal. 2:p12, 2009.

${ }^{5}$ Coskun, A. F., I. Sencan, T. Su, and A. Ozcan. Lensless wide-field fluorescent imaging on a chip using compressive decoding of sparse objects. Opt. Express 18:10510-10523, 2010.

${ }^{6}$ Coskun, A. F., T. Su, and A. Ozcan. Wide field-of-view lens-free fluorescent imaging on a chip. Lab Chip 10:824, 2010.

${ }^{7}$ Di Carlo, D., N. Aghdam, and L. P. Lee. Single-cell enzyme concentrations, kinetics, and inhibition analysis using high-density hydrodynamic cell isolation arrays. Anal. Chem. 78:4925-4930, 2006.

${ }^{8}$ Di Carlo, D., and L. P. Lee. Dynamic single-cell analysis for quantitative biology. Anal. Chem. 78:7918-7925, 2006.

${ }^{9}$ Di Carlo, D., L. Y. Wu, and L. P. Lee. Dynamic single cell culture array. Lab Chip 6:1445-1449, 2006.

${ }^{10}$ Giuliano, K. A., J. R. Haskins, and D. L. Taylor. Advances in high content screening for drug discovery. Assay Drug Dev. Technol. 1:565-577, 2003.
${ }^{11}$ Homolya, L., et al. Fluorescent cellular indicators are extruded by the multidrug resistance protein. J. Biol. Chem. 268:21493-21496, 1993.

${ }^{12}$ Hsieh, H. B., et al. High speed detection of circulating tumor cells. Biosens. Bioelectron. 21:1893-1899, 2006.

${ }^{13}$ Loo, L., et al. An approach for extensibly profiling the molecular states of cellular subpopulations. Nat. Methods 6:759-765, 2009.

${ }^{14}$ Nilsson, J., M. Evander, B. Hammarström, and T. Laurell. Review of cell and particle trapping in microfluidic systems. Anal. Chim. Acta 649:141-157, 2009.

${ }^{15}$ PDSP-MDR-1/CaCo 2 Cell Assay. < http://pdsp.med. unc.edu/pdspw/MDR.php $>$.

${ }^{16}$ Peer, D., Y. Dekel, D. Melikhov, and R. Margalit. Fluoxetine inhibits multidrug resistance extrusion pumps and enhances responses to chemotherapy in syngeneic and in human xenograft mouse tumor models. Cancer Res. 64:7562-7569, 2004.

${ }^{17}$ Perfetto, S. P., P. K. Chattopadhyay, and M. Roederer. Seventeen-colour flow cytometry: unravelling the immune system. Nat. Rev. Immunol. 4:648-655, 2004.

${ }^{18}$ Schonbrun, E., A. R. Abate, P. E. Steinvurzel, D. A. Weitz, and K. B. Crozier. High-throughput fluorescence detection using an integrated zone-plate array. Lab Chip 10:852-856, 2010.

${ }^{19}$ Stott, S. L., et al. Isolation and characterization of circulating tumor cells from patients with localized and metastatic prostate cancer. Sci. Transl. Med. 2:25ra23, 2010.

${ }^{20}$ Sun, H., E. C. Chow, S. Liu, Y. Du, and K. S. Pang. The Caco-2 cell monolayer: usefulness and limitations. Exp. Opin. Drug Metab. Toxicol. 4:395-411, 2008.

${ }^{21}$ Xia, Y., and G. M. Whitesides. Soft lithography. Angew. Chem. Int. Ed. 37:550-575, 1998.

${ }^{22}$ Young, D. W., et al. Integrating high-content screening and ligand-target prediction to identify mechanism of action. Nat. Chem. Biol. 4:59-68, 2008. 